\title{
新疆北部白冠攀雀的巢与巢址选择
}

\author{
梅 宇 ${ }^{1,2}$, 马 鸣 ${ }^{1, *}$, 胡宝文 ${ }^{1,2}$, Dusan Brinkhuizen ${ }^{3}$, Tamas Szekely ${ }^{3}$ \\ (1. 中国科学院新疆生态与地理研究所, 新疆 乌鲁木齐 $830011 ; 2$. 中国科学院研究生院, 北京 100049; \\ 3. Department of Biology and Biochemistry, University of Bath, Bath BA2 7AY, UK)
}

\begin{abstract}
摘要: 2008年4-7月, 在新疆北部对白冠攀雀巢址选择进行了研究。白冠攀雀的营巢习性特殊, 巢呈囊袋状, 结构甚为精致。对于白冠攀雀巢的研究, 采用总面积调查法, 进行地毯式的搜寻, 并结合标图法对其进行标记, 绘制分布图。研究结果共发现巢 125 个，营巢位于于临近湖泊、河流等水域附近的柳树、杨树、桦树等阔叶树上。 营巢树种以柳树为主，占 $68.80 \%$ 。巢的高度平均为 $(5.3 \pm 2.5) \mathrm{m}$ ，营巢于乔木的中下部（约 $1 / 3$ 处），约 $70 \%$ 的巢离 河边不足 $30 \mathrm{~m}$ 。对于巢址选择的研究, 将原始记录中与巢址选择有关的特征变量进行主成分分析, 分析表明, 影 响白冠攀雀巢址选择的主要因素有4种, 依次为: 郁闭度因素 (包括营巢树胸径、巢上郁闭度)、营巢树种因素(包 括营巢树种、树高、巢位高度和乔木种类)、方位因素（包括距河边距离和巢向）、食物与巢材因素。
\end{abstract}

关键词: 白冠攀雀; 巢址选择; 主成分分析; 克兰河; 乌伦古河

中图分类号：Q959.739; Q958.113 文献标识码：A＼cjkstart文章编号：0254-5853-(2009)05-0565-06

\section{Nests and Nest-site Selection of White-crowned Penduline Tit Remiz coronatus in the Northern Xinjiang}

\author{
MEI Yu ${ }^{1,2}$, MA Ming ${ }^{1, *}$, HU Bao-wen ${ }^{1,2}$, Dusan Brinkhuizen ${ }^{3}$, Tamas Szekely ${ }^{3}$ \\ (1. Xinjiang Institute of Ecology and Geography, the Chinese Academy of Sciences, Urumqi 830011, China; \\ 2. Graduate University of Chinese Academy of Sciences, Beijing 100049, China; \\ 3. Department of Biology and Biochemistry, University of Bath, Bath BA2 7AY,UK)
}

\begin{abstract}
The nest-site selection of White-crowned Penduline Tit Remiz coronatus in Northern Xinjiang was studied from April to July in 2008. It has special nesting behavior. The nest has a pocket pouch size, with a delicate structure. Research of the nest, using the method of total area investigation, included searching for nests carefully, combined with tag plotting method, and drawing the nest distribution map. In total 125 nests were found in the field, nesting on willow, poplar, birch and other hardwood trees near lakes and rivers. $68.80 \%$ of nest trees were willows Salix spp. The average height of nests was $5.3 \pm 2.5 \mathrm{~m}$. The nests were located in the lower part of arbor (about $1 / 3$ ), about $70 \%$ of nests less than 30 meters from river. Study of the nest-site selection, used a quadrate survey to investigate the parameters of nest-site characteristics. The principal component analysis indicated that there were four factors affecting the nest-site selection of the White-crowned Penduline Tit. They were: 1. canopy (including diameter of nest tree and canopy cover above nest), 2. nest tree species (including species of nest tree and arbor, height of nest tree and nest in the tree), 3. position or site (including distance to river and nest orientation ), 4. food and nest material (including canopy cover under nest).
\end{abstract}

Key words: White-crowned Penduline Tit; Nest-site selection; Principal component Analysis; Kelan River; Ulungur River

白冠攀雀（Remiz coronatus）属于雀形目 （Passeriformes）攀雀科（Remizidae）攀雀属 (Remiz)。白冠攀雀又名白顶攀雀, 体形纤小, 性
活泼，善攀援，喜倒悬在树枝上翻来翻去（ $\mathrm{Li}$ et al， 1982; Zhao, 2001)。营巢习性特殊，在水边的树枝 上编织下垂的袋状巢，属于编织巢中的瓶状巢，为 
一种十分精巧的巢型 (Tong et al, 1985; Zheng, 1995 )。白冠攀雀繁殖于新疆北部福海、阿勒泰、 青河、北屯、恰库尔图等地 (Ma, 2001; MacKinnon et al, 2000; Li et al, 1982)。白冠攀雀善于捕虫, 主 要以鳞翅目昆虫和昆虫幼虫为食, 也吃植物果实和 种子 (Ba \& Xu, 2006; Gao, 2006)。白冠攀雀属于益 鸟, 已被列入IUCN红色名录“Least Concern”物种 (IUCN，2008）及国家保护的有益的或者有重 要经济、科学研究价值的陆生野生动物名录 (国 家林业局，2000)。

栖息地在鸟类生活史中发挥着举足轻重的作 用, 其质量的优劣直接影响鸟类的分布、数量和存 活(Cody, 1985)。巢址选择是鸟类对栖息地选择的主 要组成部分, 巢址质量的优劣性程度直接影响当年 的繁殖成效(Zheng, 1995)。研究鸟类的巢址选择, 对保护和利用鸟类资源具有重要的理论和实践意 义 (Ding \& Zheng, 1997)。白冠攀雀的巢址特征和 繁殖习性见于地方性鸟类著作 ( $\mathrm{Li}$ et al, 1982; Ma, 2001; Zhao, 2001; MacKinnon et al, 2000）。Tong et al（1985）于 1982-1983 年在吉林向海自然保护区 对中华攀雀（Remiz consobrinus）繁殖生态进行了 专题报道, 但尚未有白冠攀雀巢址选择的相关研 究。笔者于 2008 年 4-7 月在新疆北部对白冠攀雀 繁殖生态和巢址选择进行了研究, 以期为该鸟的保 护提供基础生物学资料, 对丰富其物种的生活史有 着积极的意义。

\section{1 研究地区与方法}

\section{1 调查区域}

野外工作大部分于新疆北部的阿勒泰地区。调 查地点包括: 卡拉麦里、乌伦古河流域 (恰库尔图 段)、青河县、布尔根河流域（河狸保护区）、乌伦 古河流域 (冬特段)、福海县、乌伦古湖、克兰河 流域 (北屯段)、额尔齐斯河流域 (锡伯渡), 其中 调查的两个重点流域为克兰河和乌伦古河。

克兰河全长 $265 \mathrm{~km}$, 发源于阿尔泰山南坡, 属 额尔齐斯河的一条支流。北屯段河道弯曲, 河床宽 浅。两岸天然林则以杨柳科多个树种形成单优或混 交群落, 包括苦杨(Populus 1aurifolia)、额河杨( P. jrtyschensis)、黑杨(P. nigra)、银灰杨、银白杨(P. alba) 和白柳(Salix alba)等, 林下灌木种类少, 分布有阿 勒泰山楂(Crataegus ahaica)、油柴柳(S. caspica)、 毛枝柳(S. dasyclados)、灰毛柳(S. cinerea)等 (Cheng et al, 2006）。

乌伦古河以青格里河为干流, 全长 $725 \mathrm{~km}$, 位 于欧亚大陆中心, 额尔齐斯河以南, 属准噶尔干旱 区。气候特点为夏季炎热, 春早多风, 秋季凉爽, 冬季严寒漫长, 降水较少, 蒸发量大。空气干燥, 光照充足。无霜期为 110 130d (Wang et al, 2004)。 白冠攀雀在研究区内为繁殖鸟, 每年 4 月中、下旬 迁来，5-7 月为繁殖期。

\section{2 研究方法}

对于巢的研究, 采用总面积调查法。在白冠攀 雀适宜栖息地，划分若干小区，分组进行地毯式的 搜寻。对发现的每个巢进行GPS定位, 结合标图法 对其进行标记，绘制分布图（Gao et al, 2006），定 期检查各巢的状况。对于巢址选择的研究, 将按先 后所发现的巢顺序编号，记录发现巢的日期、营巢 树种等。以鸟巢为中心, 设植物调查样方, 在每个 样方内测量并计算以下数据: 营巢树高、乔木棵数、 乔木高度 ( $\mathrm{m}$, 样方内所有乔木的平均高度)、乔木 胸径 $(\mathrm{cm}$, 乔木的平均胸径)、巢位高度、筑巢阶 段 (巢最终阶段)、巢向、巢上和巢下郁闭度、巢 距河边距离等(Wang et al, 2007)。其中巢向指巢相对 于树干的方向, 取值为以树干为轴, 自正北方向顺 时针旋转至巢所转过的度数（Li et al, 2001）。简洁 描述发现时的状况和其他任何有价值的内容, 同时 记录栖息地的植被结构及利用状况。

对原始记录中与巢址选择有关的特征变量进 行主成分分析(principal component analysis), 提取特 征值大于 1 的成分作为主成分, 确定白冠攀雀对巢 址选择的主要因素（Ding \& Zheng, 1997; Ding et al, 2008; Wang et al, 2007）。用Shapiro-Wilk test检验巢 高/营巢树高是否符合正态分布，符合正态分布用 单组设计定量资料的 $t$-检验分析, 检验巢的高度是 否在营巢树高的中下部。数据用SAS 9.0 统计软件 分析处理。文中数据用平均值土标准误表示。

\section{2 结 果}

\section{1 巢与营巢}

白冠攀雀巢呈囊袋状, 主要由柳絮、杨絮等编 织而成, 结构甚为精致。巢顶端侧面有一管状开孔, 供亲鸟出入 (图 1)。白冠攀雀营巢于湖泊、河流等 水域附近的柳树、杨树、桦树等阔叶树上，营巢始 于 4 月下旬, 5 月达到高峰, 至 7 月上旬结束。共 发现 125 个巢, 营巢树以柳树为主。86 个营巢于柳 
树上 $(68.80 \%) ; 37$ 个营巢于杨树上 $(29.60 \%) ; 2$ 个营巢于桦树上 $(1.60 \%)$ 。野外观察, 白冠攀雀营 巢很快, 整个过程 7 $8 \mathrm{~d}$ 即可完成。

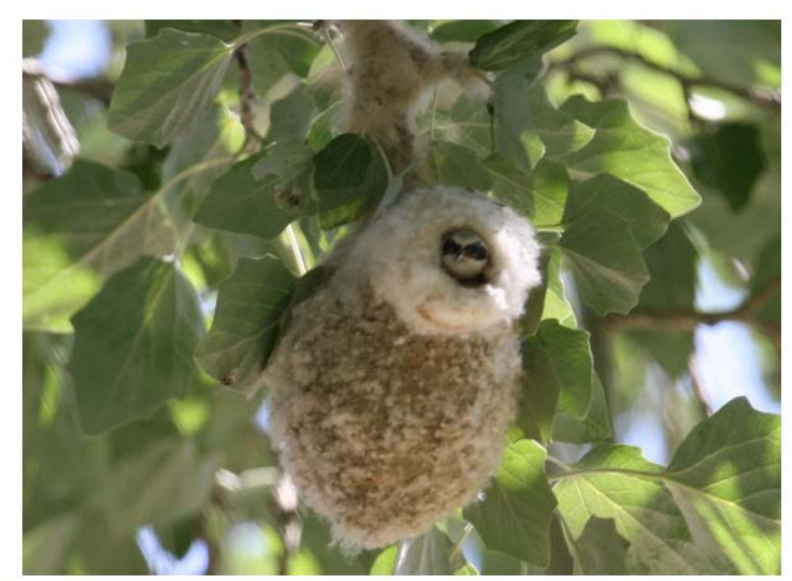

图 1 白冠攀雀及巢

Fig. 1 The nest of White-crowned Penduline Tit

\section{2 巢高与营巢树高}

巢高平均为 $(5.3 \pm 2.5) \mathrm{m}, 46.67 \%$ 的巢高在 $4 \sim 6$ $\mathrm{m}$ 之间。营巢树高在5 30 m。柳树较低, 大多 $15 \mathrm{~m}$ 以下, 平均为 $(13.7 \pm 4.7) \mathrm{m}(n=86)$, 桦树较高, 平均 $(25.0 \pm 0) \mathrm{m}(n=2)$, 杨树可高达 $30 \mathrm{~m}$, 平均 为 $(20.3 \pm 6.2) \mathrm{m}(n=37)$ 。其巢位于营巢树的位置 相当，在其树高的中下部 $(32 \% \sim 40 \%)$ 。用 Shapiro-Wilk test检验巢高/营巢树高符合正态分布,

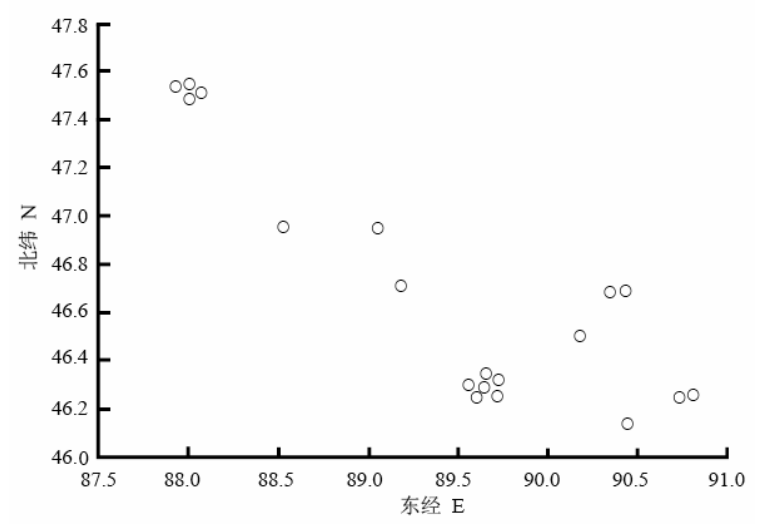

图 3 乌伦古河 (冬特段) 巢点分布 $(n=19)$

Fig. 3 Nest distribution of Ulungur River (Dongte)

\section{5 巢址选择主成分分析}

对营巢树种、营巢树高、乔木胸径、巢位高度、 筑巢阶段、巢上和巢下郁闭度、巢距河边距离等11 个巢址特征变量进行主成分分析的结果显示, 前4 个成分的特征值大于 1 , 提取为主成分, 这 4 个主成 $t$-检验分析结果也证实其巢约在营巢树高的 $1 / 3$ 处 （图2）。

\section{3 距河边距离}

巢距河边的平均距离为 $(27.3 \pm 27.3) \mathrm{m}(n=117)$, 有 24 个悬于水面上方, $69.57 \%$ 的巢离河边不到 30 $\mathrm{m}$, 最远距河边 $120 \mathrm{~m}$ 。

\section{4 巢空间分布}

调查的两个重点流域为乌伦古河（冬特段）和 克兰河 (北屯段)。利用GPS记录每个巢点的经纬度, 绘制出分布图3、图4。从图中可以看出, 巢点分布 较集中，沿河流的两岸河谷林分布。巢间距离最近 仅 $3 \mathrm{~m}$, 巢密度可达 24.9 个 $/ \mathrm{km}^{2}$ (克兰河)。白冠攀 雀有集群营巢倾向。

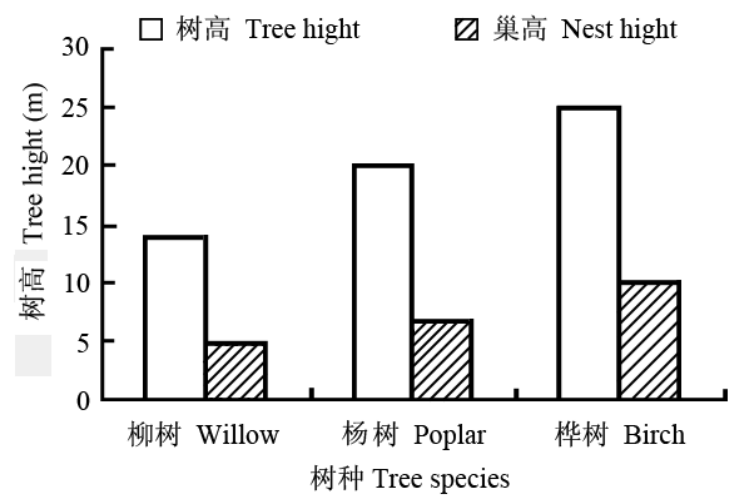

图 2 三种巢与营巢树平均高度 $(n=117)$

Fig.2 The average height between nest and tree

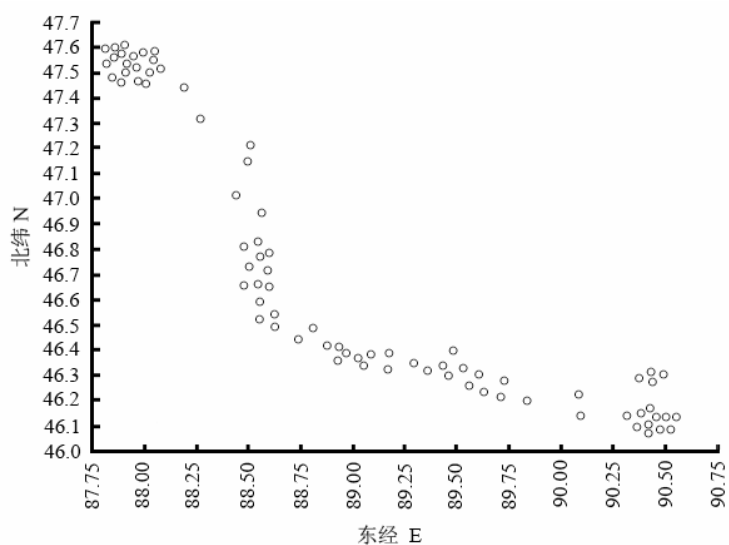

图 4 克兰河（北屯段）巢点分布 $(n=78)$

Fig. 4 Nest distribution of Kelan River (Beitun)

分的方差对总方差的累计贡献率为 $67.93 \%$ (表1)。说 明前 4 个主成分基本包含了巢址参数所具有的信 息, 足以解释白冠攀雀对巢址的选择性。因此提取 这 4 个主成分并计算它们与原始变量的因子载荷, 结果见表2。 
由表2和表3知, 第一主成分中, 乔木胸径、巢 上郁闭度的载荷最大, 反映了两者成正相关, 因此 定为郁闭度因素。第二主成分中, 营巢树种、营巢 树高、乔木种类、巢位高度的载荷较大, 反映了营 巢树的特征, 将其定为营巢树种因素。第三主成分
中, 距河边距离和巢向的载荷最大, 距河边距离的 载荷为正值, 巢向的载荷为负值, 反映了栖息环境 与避风条件，将其定为方位因素。第四主成分中， 巢下郁闭度的载荷最大, 反映了食物与巢材条件, 因此将其定为食物与巢材因素。

表 1 白冠攀雀巢址选择主成分分析 $(n=75)$

Tab. 1 Analysis of the component of nest-site selection of Remiz coronatus

\begin{tabular}{|c|c|c|c|c|}
\hline 主成分 component & 特征值 Eigenvalues & Ratio of variance & 累计贡献率 (\%) & Accumulative ratio of contribution \\
\hline 1 & 2.629 & 23.900 & & 23.900 \\
\hline 2 & 2.454 & 22.310 & & 46.210 \\
\hline 3 & 1.322 & 12.020 & & 58.220 \\
\hline 4 & 1.067 & 9.700 & & 67.930 \\
\hline 5 & 0.915 & 8.320 & & 76.250 \\
\hline 6 & 0.757 & 6.890 & & 83.130 \\
\hline 7 & 0.654 & 5.940 & & 89.080 \\
\hline 8 & 0.481 & 4.370 & & 93.440 \\
\hline 9 & 0.352 & 3.200 & & 96.640 \\
\hline 10 & 0.210 & 1.910 & & 98.550 \\
\hline 11 & 0.159 & 1.450 & & 100.00 \\
\hline
\end{tabular}

表 2 巢址选择参数特征向量的转置矩阵

Tab. 2 Matrix of nest-site selection factors

\begin{tabular}{|c|c|c|c|c|}
\hline \multirow{2}{*}{ 变量Variable } & \multicolumn{4}{|c|}{ 特征向量Total vector } \\
\hline & 1 & 2 & 3 & 4 \\
\hline 营巢树种 Nest tree species & -0.195 & 0.503 & -0.061 & 0.255 \\
\hline 巢位高度 Height of nest in the tree (m) & 0.158 & 0.430 & 0.284 & 0.027 \\
\hline 下垂树枝Droping branches (cm) & 0.269 & -0.082 & 0.261 & -0.247 \\
\hline 乔木胸径 Diameter of nest (cm) & 0.538 & 0.110 & -0.123 & -0.085 \\
\hline 巢上郁闭度 Canopy cover above nest ( \%) & 0.524 & 0.072 & -0.103 & -0.025 \\
\hline 巢下郁闭度Canopy cover under nest ( \%) & -0.046 & -0.123 & 0.332 & 0.768 \\
\hline 乔木数量 Number of arbor & -0.375 & 0.181 & -0.318 & -0.220 \\
\hline
\end{tabular}

表 3 巢址选择主成分分类及命名

Tab. 3 Classification and nomination of nest-selection factors

\begin{tabular}{|c|c|c|c|c|}
\hline 主成分 & 参数 & 平均值 & 命名 & 贡献率 \\
\hline Main factors & Parameter & Average value & Name of factor & Ratio of contribution \\
\hline \multirow{2}{*}{1} & 营巢树胸径 Diameter of nest tree (cm) & $36.6 \pm 26.8$ & \multirow[t]{2}{*}{ 郁闭度因素 Factor of canopy } & \multirow[t]{2}{*}{23.900} \\
\hline & 巢上郁闭度Canopy cover above nest $(\%)$ & $54.8 \pm 13.2$ & & \\
\hline \multirow{4}{*}{2} & 营巢树种 Nest tree species & & & \multirow{4}{*}{22.310} \\
\hline & 营巢树高 Height of nest tree (m) & $16.1 \pm 5.4$ & 营巢树种因素 & \\
\hline & 巢位高度 Height of nest in the tree (m) & $5.1 \pm 2.1$ & Factor of nest trees species & \\
\hline & 乔木种类 Species of arbor & & & \\
\hline \multirow{2}{*}{3} & 距河边距离Distance to river (m) & \multirow{2}{*}{$27.2 \pm 27.3$} & \multirow{2}{*}{ 方位因素 Factor of orientation } & \multirow{2}{*}{12.020} \\
\hline & 巢向Nest orientation $\left(^{\circ}\right)$ & & & \\
\hline \multirow{2}{*}{4} & \multirow{2}{*}{ 巢下郁闭度Canopy cover under nest (\%) } & \multirow{2}{*}{$56.8 \pm 18.4$} & 食物与巢材因素 & \multirow{2}{*}{9.700} \\
\hline & & & Factor of food and nest material & \\
\hline
\end{tabular}

3 讨 论

巢址选择是鸟类繁殖的重要环节, 能将同类的 干扰作用、天敌捕食和不良因子的影响降低到最低
水平, 从而提高繁殖成功率 (Lack, 1969; Ding et al, 2008）。通过选择巢址, 鸟类能够找到一个相对适 宜的繁殖地点以保证繁殖活动顺利进行。在这种选 
择中, 许多鸟类都倾向于选择那些能使其繁殖成效 最大而存活代价最小的营巢生境 (Ding \& Zheng, 1997）。由于栖息地中的各种因子对于动物的作用 不同, 分析其内在特征, 找出影响选择的主要因子 是研究的关键所在(Gao et al, 2006)。

白冠攀雀营巢时间与杨柳开花吐絮的旺季相符。由 于无脊椎动物的丰富度与灌木、草本层植物的发育 状况相关 (Tscharntke \& Greiler, 1995; Morris \& Thompson, 1998; Ding et al, 2008）, 而白冠攀雀主 要以昆虫为食, 故推测其营巢时间的选择可能与食 物的丰盛度有关。实地调查也证实 4-7 月蚊子等 昆虫最为丰富, 为其提供了充足的食物。Tong et al (1985) 将营巢程序分为扎框、织篮、封窗、造管 4 个阶段。Rene et al (2007) 将欧亚攀雀 (Remiz pendulinus）营巢分为 6 个阶段, 两者大同小异。

白冠攀雀对巢高具有选择性, 均营巢于乔木的 中下部（图2）, 巢下灌木较少。乔木中下部郁闭 度高、通透性好, 便于进出巢。位置的特殊性可以 使育倠期的倠鸟免受大风、干燥、炎热等恶劣气候 的影响, 从而保证其存活。

乔木高度和灌木密度等植被因子是影响白冠 攀雀巢址选择的重要因素。白冠攀雀利用的巢树均 较为高大 $(13.7 \pm 4.7 \mathrm{~m})]$, 且周围的乔木也较为高 大, 生境中高度相对均一的乔木可能会为白冠攀雀 营巢提供更多的选择。通过野外观察发现, 白冠攀 雀喜欢飞到河边的灌从采集巢材, 林下灌木层的稀 疏有利于其在林中穿梭飞行, 而巢下郁闭度高则会 抑制其飞行。

距水源距离也是影响白冠攀雀对巢址进行选 择的一个重要因素。水是动物生活所必需的资源物 质, 也是其最重要的生存条件之一 (Sun, 2001; Gao

\section{参考文献:}

Ba GL, Xu X. 2006. The nest of penduline tit: A valuable Chinese traditional medicinal materials is used by local Kazak people in Altay [J]. J Chn Med Mat, 29(7): 658. [巴哈尔古丽, 徐 新. 2006. 灵 雀窝阿勒泰地区哈萨克民间应用的一种名贵中药材. 中药材, 29 (7): 658.]

BirdLife International. 2008. Remiz coronatus [EB/OL]. IUCN 2008. 2008 IUCN Red List of Threatened Species. www.iucnredlist.org.

Cody. 1985. Habitat Selection in Birds[M]. New York: Academic Press, 4-9.

Cheng KW, Zang RG, Zhou XF, Zhang WY, Bai ZQ. 2006. Influence of flood on the natural riparian forests in the area of the Ergis River[J]. $J$ Beijing Forest Univ, 28(2): 46-51. [成克武, 藏润国, 周晓芳, 张炜银, 白志强. 2006. 洪水对额尔齐斯河河岸天然林植被的影响研究. 北 et al, 2006）。研究区内的水源多为季节性河流，白 冠攀雀的巢距离水源较近, 约 70\%的巢离河边不足 $30 \mathrm{~m}$ 。水源并非直接影响白冠攀雀的生存, 但为其 提供了良好的生境与食物, 可以满足成鸟在繁殖期 的需求, 从而为其繁殖成功提供保障。

主成分分析可以从多指标的复杂事物中找出 生命活动的主要倾向, 帮助人们了解生命活动的规 律和本质(Ding \& Zheng, 1997)。根据白冠攀雀巢址 选择有关的 11 项指标进行的主成分分析，第一个 和第二个主成分的贡献率较高 (23.90\%和 $22.31 \%$ ), 说明“郁闭度因素”和“营巢树种因素”在白冠攀雀 的巢址选择中处于重要地位。由表 2 中的特征向量 值可知, 白冠攀雀倾向于选择粗大的树木营巢, 营 巢树以柳树为主 $(68.80 \%)$, 这与河谷林的优势种 相符。巢的位置在营巢树种的中下部且巢上方盖度 较大。巢上郁闭度大, 可有效避免强风、降雨等自 然灾害。第三个主成分“方位因素” 的贡献率略低 $(12.02 \%)$, 占有较重要的地位, 由于巢向的特征值 为负值(表 2), 说明白冠攀雀喜爱在靠近河流且避风 的一侧营巢 $(70.67 \%)$ 。第四个主成分“食物与巢 材因素”的贡献率较低 $(9.70 \%)$, 但它的特征值较高, 也有重要的地位, 反映出白冠攀雀倾向于在河岸两 边的柳枝灌从获取昆虫等食物和柳絮等巢材, 这与 实际观察的结果是一致的。

致谢: 本研究得到英国巴斯大学等的技术和经 费支持。感谢中国科学院新疆生态与地理研究所及 新疆观鸟会张国强、王春芳、苟军、夏咏、戴江南、 李维东、徐捷、段士民、R. Dijk（荷兰）、A. Pogány (英国)、陈莹、徐峰、吴逸群等的参与和帮助。

京林业大学学报, 28(2): 46-51.]

Ding CQ, Zheng GM. 1997. The Nest Site Selection of The Yellow -Bellied Tragopan (Tragopan caboti) [J]. Acta Zool Sin, 43(1): 27-33.[丁长青, 郑光美. 1997. 黄腹角雉的巢址选择. 动物学报, 43(1): 27-33.] Ding ZF, Yuan L, Zhu CL, Huang JW, Zheng XQ, Guo XP, Lin JY, Hu HJ, Tang SX. 2008. Nesting characteristics of Yellow-bellied Prinia Prinia flaviventris[J]. Zool Res, 29(3): 270-276. [丁志锋, 袁 玲, 朱成林, 黄进文, 郑细群, 郭向品, 林杰妤, 胡慧建, 唐思贤. 2008. 黄腹山 鹪莺的营巢特征. 动物学研究, 29(3): 270-276.]

Gao W. 2006. Studies on Birds and their Ecology in Northeast China[M]. Beijing: Science Press. [高 玮. 2006. 中国东北地区鸟类及其生态 学研究. 北京: 科学出版社, 257.]

Gao ZJ, Du ZY, Wang XS, Huang H, Wang K, Yang CB. 2006. The 
nest-site selection of Spangled Drongo at Dongzhai Nature Reserve,Henan[J]. Chn J Zool, 41(1): 69-73. [高振建, 杜志勇, 王兴森, 黄 华, 王 科, 杨春柏. 2006. 河南董寨国家级自然保护区发冠 卷尾的巢址选择. 动物学杂志, 41(1): 69-73.]

Lack D. 1969. The number of bird species on island [J]. Bird Study, 16: 193-209.

Li GY, Zheng BL, Liu GZ. 1982. Fauna Sinica: Vol.13: Passeriformes [M] Beijing: Science Press, 99-102. [李桂垣, 郑宝㐘, 刘光佐. 1982. 中国 动物志. 鸟纲: 第十三卷, 雀形目 (山雀科一绣眼鸟科). 北京: 科学 出版社, 99-102.]

Li XH, Ma ZJ, Li DM, Ding CQ, Zhai TQ, Lu BZ. 2001. Using resource selection functions to study nest site selection of Crested Ibis[J]. Biodivers Sci, 9(4): 352-358. [李欣海, 马志军, 李典谟, 丁长青, 翟 天庆, 路宝忠. 2001. 应用资源选择函数研究朱睘的巢址选择. 生 物多样性, 9(4): 352-358.]

Ma M. 2001. A checklist of the birds in Xinjiang, China[J]. Arid Zone Res, 18 (Suppl.): 1-90.[马鸣. 2001. 新疆鸟类名录. 干早区研究, 18 (增 刊 ) : 1-90.]

MacKinnon J, Phillipps K, He FQ. 2000. A Field Guide to the Birds of China[M]. Changsha: Hunan Education Press, 324.[约翰·马敬能, 卡 伦.菲力普斯, 何芬奇. 2000. 中国鸟类野外手册. 长沙: 湖南教育 出版社, 324.]

Morris DL, FR Thompson III. 1998. Effects of habitat and invertebrate density on abundance and foraging behavior of brown-headed cowbirds [J]. Auk, 115(2): 376-385.

Van Dijk, Rene E, Szentirmai I, Szekely T. 2007. Practical Field Guide for Mvestigating Breeding Ecology of Penduline Tit Remiz pendulinus.
[M]. [Bath]:[University of Bath]

Sun RY. 2001. Principles of Animal Ecology [M].3rd ed. Beijing: Beijing Normal University Press, 71.[孙儒泳. 2001. 动物生态学原理..3 版. 北京：北京师范大学出版社, 71.]

Tong JC, Zhou WW, Yang XM, Jiang ML. 1985. Studies on the breeding ecology of the Penduline Tit[J]. Acta Zool Sin 31(2): 154-161. [童骏昌, 周薇薇, 杨学明, 姜牧里. 1985. 攀雀繁殖生态的研究. 动物学报, 31(2): 54-161.]

Tscharntke T, Greiler H. 1995. Insect communities, grasses, and grasslands [J]. Ann Rev Entomol, 40: 535-558.

Wang N, Zhang YN, Zheng GM. 2007. Nest and nest-site of Narcissus Flycatcher in Xiaolongmen Forestry, Beijing[J]. Zool Res, 28(4): 337-343. [王 宁, 张雁云, 郑光美. 2007. 北京小龙门林场黄眉姬暡 的巢与巢址特征. 动物学研究, 28(4): 337-343.]

Wang ZS, Cheng TF, Liu KH, Jiang HM. 2004. Water resources and its features in Ulungur River watershed,Xinjiang[J]. Arid Land Geogry, 23(2): 123-126. [王振升, 程同福，刘开华，蒋惠敏. 2004. 乌伦古河 流域水资源及其特征. 干旱区地理, 23(2): 123-126.]

Zhao ZJ. 2001. Chinese Avifauna.: Vol. II : Passeriformes [M]. Changchun: Jilin Science and Technology Press, 741-745. [赵正阶. 2001. 中国岛 类志: 下卷. 长春: 吉林科学技术出版社, 741-745.]

Zheng GM. 1995. Ornithology[M]. Beijing: Beijing Normal University Press.[郑光美. 1995. 鸟类学. 北京: 北京师范大学出版社.]

Zheng GM. 2005. A Checklist on the Classification and Distribution of the Birds of China [M]. Beijing: Science Press.[郑光美. 2005. 中国鸟类 分类与分布目录. 北京: 科学出版社.] 